УДК $532.5 .013 .12 / 536.423 .1$

\title{
ПОТЕРИ ДАВЛЕНИЯ ПРИ ВЫНУЖДЕННОМ ТЕЧЕНИИ ВОДЫ В ПАРОГЕНЕРИРУЮЩИХ КАНАЛАХ
}

\author{
Антипов B.Г. \\ Научно-производственное предприятие "ПАСКАЛЬ", ул. Желябова, 2а, Киев, 03680, Украина \\ antipov.paskal@gmail.com
}

На основі отриманих експериментальних даних пропонуються співвідношення для визначення втрати тиску для двофазної течії води у вертикальній трубі та каналі у вигляді кільця в умовах теплопідведення. Дослідження проведені при тиску від 4 до 16 МПа, масовій швидкості від 300 до $3000 \kappa г /\left(\mathrm{M}^{2} \mathrm{c}\right)$, тепловому потоці до 2,4 $\mathrm{MBT} / \mathrm{M}^{2}$. Результати досліджень можуть бути використані для теплогідравлічних розрахунків елементів енергетичних установок.
На основе полученных экспериментальных данных предлагаются соотношения для определения потерь давления при двухфазном течении воды в вертикальной трубе и кольцевом канале в условиях теплоподвода. Исследования проведены при давлении от 4 до 16 МПа, массовой скорости от 300 до $3000 \mathrm{\kappa г} /\left(\mathrm{M}^{2} \mathrm{c}\right)$, тепловом потоке до $2,4 \mathrm{MBT} / \mathrm{M}^{2}$. Результаты исследований могут использоваться для теплогидравлических расчетов элементов энергетических установок.
Relations for the pressure drop of the two phase flower of water in vertical heater pipe and annular channel are proposed. The study was carried out at a pressure of from 4 to $16 \mathrm{MPa}$, mass velocity from 300 to $3000 \mathrm{~kg} /\left(\mathrm{m}^{2} \mathrm{~s}\right)$, heat is the current up to $2.4 \mathrm{MW} / \mathrm{m}^{2}$. The obtained results can be used for thermal and hydraulic calculation of elements of power plants.

Библ. 19, табл. 1, рис. 9.

Ключевые слова: двухфазный поток, потери давления, вертикальная труба, кипение.

При расчете потерь давления в парогенерирующих каналах используются корреляционные соотношения, полученные на основе экспериментальных исследований. Эмпирические зависимости, которые предлагаются в справочниках [1-3] и других публикациях, в самом общем виде не противоречат характеру поведения гидравлического сопротивления, но имеют существенные ограничения по применению и часто расходятся по количественной оценке.

Сложилось несколько подходов к описанию потерь давления при течении двухфазного потока в каналах. Широкое распространение получил метод расчета с использованием параметров двухфазности, которые предложены Martinelli R.C. с соавторами [4]. Метод основан на рассмотрении потерь давления при раздельном течении фаз в зависимости от сочетания режимов течения в каждой из них. Для определения параметров двухфазности используются эмпирические номограммы.

В работах [5-9] авторы предлагают корреляционные соотношения для относительных потерь давления $\psi$, которые представляют собой отношение потерь при движении двухфазной смеси $\Delta P$ к потерям при движении насыщенной жидкости $\Delta P_{0}$. При этом функция $\psi$ (или коэффициент двухфазности) объединяет влияние всех основных характеристик - физических свойств, массовой скорости, давления, теплового потока и паросодержания. В работах [7-9] на основании опытных данных, полученных в ЦКТИ [5], приведены достаточно громоздкие уравнения для относительных потерь давления.

Авторы работ $[10,11]$ предложили иной метод обоб- щения экспериментальных данных. Он за-ключается в разложении коэффициента гидравлического сопротивления на произведение независи-мых функций от влияющих параметров. Следуя Боришанскому В.М. [10], потери давления в канале с двухфазным потоком можно выразить уравнением:

$$
\Delta P_{2 \Phi}=\Delta P-\Delta P_{0}=F(x) F(\rho w) F(P) F(q) F(D) \frac{(\rho w)^{2}}{2 \rho_{l s}} \frac{\Delta Z}{D} .
$$

Здесь $\Delta P$ - потери давления на участке канала $\Delta Z \mathrm{c}$ гидравлическим диаметром $D, F(x)$ - некоторая функция от паросодержания, $F(\rho w), F(P), F(q), F(D)-$ функции влияния массовой скорости $\rho w$, давления $P$, теплового потока $q$, и геометрии канала, соответственно, $\Delta P_{0}-$ потери давления для однофазного турбулентного течения насыщенной жидкости:

$$
\Delta P_{0}=\xi_{0} \frac{(\rho w)^{2}}{2 \rho_{l s}} \frac{\Delta Z}{D},
$$

где $\xi_{0}$ - коэффициент гидравлического сопротивления, $\rho_{l s}-$ плотность жидкости при температуре насыщения.

Форма уравнения (1) может быть использована в том случае, если влияние параметров на потери давления могут быть определены экспериментально с достаточной точностью. В работе [10] описаны в явном виде функции от паросодержания, давления, массовой скорости и относительного шага в пучке стержней. Влияния теплового потока на потери давления в этой работе обнаружено не было $(F(q)=1)$ в отличие от результатов других авторов. Неоднозначность в оценке влияния теплоподвода, по-видимому, связана с большими погрешностями опытов, поскольку противоречивость выводов 
обнаруживается у одних и тех же авторов. В работе [5] тепловой поток введен в корреляционные соотношения в виде степенной функции $q^{0.7}$, а в работе $[6]-F(q) \sim q^{1}$.

Доказанной можно считать зависимость потерь давления от массовой скорости в виде степенной функции, несмотря на то, что показатель степени у разных авторов отличается от 1.3 до 1.6. На основании экспериментальных данных, полученных в газожидкостных и пароводяных восходящих потоках в адиабатных условиях и в условиях с теплоподводом, Silvestri M. [12] определяет это влияние степенной функцией с показателем 1.4. Такой же результат получен в исследованиях Боришанского В.М. с соавторами на пучках стержней [10]. Крайние значения показателя степени для двухфазного потока с подводом тепла встречаются в работе [5] и в работах авторов из CISE (Италия) [12], где функции представлены, соответственно, как $(\rho w)^{1.3}$ и $(\rho w)^{1.6}$.

Характер поведения потерь давления в зависимости от паросодержания в упомянутых работах описан поразному. В ранних работах $[5,6]$ представлены опытные данные, которые предполага-ют существование $F(x)$ с двумя экстремумами в широком диапазоне изменения массовой скорости. Положение экстремумов относительно оси паросодержания в исследованиях не являлось фиксиро ванным, а зависело от массовой скорости и давления. В работе [13] предпринята попытка объяснить аномальное поведение гидравлического сопротивления развитием волнового движения на поверхности пристенной пленки жидкости. Там же предполагается существование роста гидравлического сопротивления после его падения вследствие исчезновения волн на поверхности пленки. Обоснование приведено для адиабатного потока, но отсутствует для течения в канале с теплоподводом. В других исследованиях $[4,10]$ авторы склоняются к описанию $F(x)$ как непрерывной функции с одним максимальным значением при некотором достаточно высоком паросодержании.

В настоящей работе представлена методика расчета потерь давления, основанная на экспериментальном доказательстве возможности использования уравнений типа (1).

\section{Экспериментальное оборудование}

Экспериментальные исследования проведены на замкнутом циркуляционном контуре высокого давления Института технической теплофизики (г. Киев). В качестве рабочего участка использовались цельнотянутые трубы из нержавеющей стали $12 \mathrm{X} 18 \mathrm{H} 10$ Т для трубных каналов с наружным диаметром 0.016 м (при толщине стенки 0.0015 м) и 0.014 м (при толщине стенки 0.002 м), а для кольцевых - трубы с наружным диаметром 0.04 и 0,032 м для образования кольцевой щели 0,002 м. Рабочий участок обогревался постоянным электрическим током. Отборы давления были выполнены в виде щели шириной $2 \cdot 10^{-4}$ м. Перепад давления измерялся с помощью быстродействующего преобразователя дифференциального давления, выходной сигнал которого передавался на ПК. В режиме реального времени архивировались сигналы от преобразователей тока, напряжения, температуры, перепада давления и расхода. В качестве теплоносителя использовалась обессоленная вода. В таблице представлен диапазон изменения режимных параметров при проведении исследований.

Табл. Геометрические и режимные параметры при исследовании гидравлического сопротивления

\begin{tabular}{|c|c|c|c|c|c|c|c|}
\hline Геометрия & $\begin{array}{c}\text { Длина из- } \\
\text { меритель- } \\
\text { ного } \\
\text { участка, } \\
\text { м }\end{array}$ & $\begin{array}{c}\text { Давле- } \\
\text { ние, МПа }\end{array}$ & 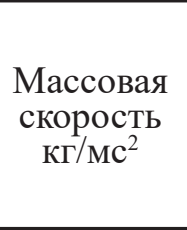 & $\begin{array}{l}\text { Тепловой } \\
\text { поток, } \\
\text { МВт/м² }\end{array}$ & $\begin{array}{c}\text { Среднее } \\
\text { паросо- } \\
\text { держание } \\
\text { на измери- } \\
\text { тельном } \\
\text { участке, \% }\end{array}$ & $\begin{array}{c}\text { Коли- } \\
\text { чество } \\
\text { режимов }\end{array}$ & $\begin{array}{c}\text { Средняя } \\
\text { ошибка } \\
\text { резуль- } \\
\text { татов } \\
\text { опыта } \\
\text { \% }\end{array}$ \\
\hline $\begin{array}{c}\text { Труба } \\
L_{h}=2,835 \\
D=0,01\end{array}$ & 0,635 & 7, 1014 & $265 \ldots 3000$ & $0,12 \ldots 2,4$ & $0 \ldots 87$ & 21 & 25 \\
\hline $\begin{array}{c}\text { Труба } \\
L_{h}=2,66 \\
D=0,013\end{array}$ & $\begin{array}{c}0,975 \\
0,65 \\
0,325\end{array}$ & $\begin{array}{l}4,6,8 \\
12,14\end{array}$ & $300 \ldots 1200$ & $0,26 \ldots 1,48$ & $0 \ldots 83$ & 31 & 25 \\
\hline $\begin{array}{c}\text { Кольцевой } \\
\text { канал } \\
D=0,004 \\
\text { односторон- } \\
\text { ний обогрев }\end{array}$ & 0,56 & $4,6,10$ & $300 \ldots 1000$ & $0,3 \ldots 1,5$ & $0 \ldots .21$ & 7 & 25 \\
\hline
\end{tabular}

$L_{h}$ - обогреваемая длина участка, м; $D$ - гидравлический диаметр, м.

\section{Потери давления в вертикальном канале}

Необратимые потери давления для двухфазного потока в канале экспериментально можно определить по измерению перепада давления между выбранными сечениями. При обработке данных применяют как урав- нение сохранения энергии, так и уравнение сохранения количества движения. Последнее чаще всего используют в публикациях, когда результаты измерений представляют в виде потерь давления на трение со стенкой. Предполагая существование квазигомогенного течения, 
для которого теплофизические свойства смеси осреднены по сечению, а скорости фаз неодинаковы, уравнение движения для вертикального канала можно записать в виде [14]:

$$
-\frac{d P}{d Z}=\xi \frac{(\rho w)^{2}}{2 \rho^{*} D}+(\rho w)^{2} \frac{d}{d Z}\left[\frac{x^{2}}{\varphi \rho_{g}}+\frac{(1-x)^{2}}{(1-\varphi) \rho_{l}}\right]+g \rho_{m},
$$

где $x, \varphi$ - массовое и объемное паросодержание, $\rho_{g}, \rho_{l}$, $\rho_{m}-$ плотность газа, жидкости и смеси, соответственно, $\rho^{*}$ - плотность, которая будет определена в дальнейшем, $\xi$ - коэффициент гидравлического сопротивления.

Измерительный участок обычно располагают в выходной части обогреваемой трубы для того, чтобы получить данные для максимальных значений паросодержания смеси. В общем случае участок $\Delta Z$ может охватывать две характерные области: область неразвитого кипения при термодинамически неравновесном течении $\Delta Z_{1}$ и область развитого кипения в равновесном потоке $\Delta Z_{2}$. В зависимости от положения отборов давления относительно этих областей изменяется и алгоритм обработки опытных данных. Потери давления на трение в вертикальном измерительном участке $\Delta Z=\Delta Z_{1}+\Delta Z_{2}$ представим в виде суммы:

$$
\begin{aligned}
& \Delta P=\Delta P_{\text {exp }}-(\rho w)^{2}\left[\left(\frac{x^{2}}{\varphi \rho_{g}}+\frac{(1-x)^{2}}{(1-\varphi) \rho_{l}}\right)_{\text {out }}-\left(\frac{x^{2}}{\varphi \rho_{g}}+\frac{(1-x)^{2}}{(1-\varphi) \rho_{l}}\right)_{\text {in }}\right]+\varepsilon \\
& +g \Delta Z\left[\rho_{\infty}-\frac{1}{\Delta Z}\left(\left\langle\rho_{1}\right\rangle \Delta Z_{1}+\left\langle\rho_{2}\right\rangle \Delta Z_{2}\right)\right],
\end{aligned}
$$

где $\Delta P_{\text {ехр }}$ - перепад, измеренный преобразователем разности давления, $\rho_{\infty}-$ плотность воды в линиях отбора давления, $\left\langle\rho_{1}\right\rangle-$ средняя плотность смеси на участке $\Delta Z_{1},<\rho_{2}>-$ средняя плотность смеси на участке $\Delta Z_{2}$, индексы in, out относятся ко входу и выходу измерительного участка. Среднюю плотность по длине выбранного участка можно определить интегрированием:

$$
\left\langle\rho_{k}\right\rangle=\frac{1}{\Delta \varphi_{k}} \int \rho_{k}\left(\varphi_{k}\right) d \varphi, k=1,2,
$$

где $k$ - индекс области интегрирования в соответствии с (2). По относительной энтальпии $x_{r, j}=\left(h_{j}-h_{l s}\right) / r$ в $j$-м сечении внутри области интегрированния можно определить массовое $x_{j}$ и объемное $\varphi_{j}$ паросодержания по формулам из работы [15]. Энтальпию пароводяной смеси $h_{j}$ на расстоянии $Z_{j}$ от начала обогрева определим по тепловому балансу без учета потерь как $h_{j}=h_{i n}+(4 q /$ $\rho w)(Z / D)$. Для участка с неразвитым кипением при $x_{i} \leq x_{r} \leq x_{e}$ получим: $\dot{\rho}_{j}=\left(1-\varphi_{j}\right) \rho_{l, j}+\varphi_{j} \rho_{g s}$

$$
\begin{gathered}
\varphi_{j}=\left[1+\frac{1-x_{j}}{x_{j}} s_{j} \frac{\rho_{g}}{\rho_{l}}\right]^{-1} ; x_{j}=x_{e}\left(1-X_{j}\right) \exp \left[\frac{x_{i}}{x_{e}} X_{j}\right] ; X_{j}=\frac{x_{e}-x_{r, j}}{x_{e}-x_{i}} ; \\
x_{i}=-370(1+0.63 \pi) N_{b} ; x_{e}=71(1-0.065 \pi) N_{b} ; \\
s_{j}=1+\left(0.6+1.5 \beta_{j}^{2}\right)(1-\pi) \mathrm{Fr}^{-0.25} ;
\end{gathered}
$$

$$
\beta_{j}=\left(1+\frac{\rho_{g}}{\rho_{l}} \frac{\left(1-x_{j}\right)}{x_{j}}\right)^{-1} ; \mathrm{Fr}=\frac{(\rho w)^{2}}{\rho_{l s}^{2} g D}
$$

Для участка с развитым кипением при $x_{e} \leq x_{r} \leq 1$ :

$$
\begin{gathered}
\rho_{j}=\left(1-\varphi_{j}\right) \rho_{l s}+\varphi_{j} \rho_{g s}, \\
\varphi_{j}=\left[1+\frac{1-x_{j}}{x_{j}} s_{j} \frac{\rho_{g}}{\rho_{l s}}\right]^{-1}, x_{j}=x_{r, j}, \beta_{j}=\left(1+\frac{\rho_{g}}{\rho_{l s}} \frac{\left(1-x_{j}\right)}{x_{j}}\right)^{-1} .
\end{gathered}
$$

В этих соотношениях: $x_{i}, x_{e}$ - относительная энтальпия в сечении начала кипения и в сечении перехода к развитому кипению, соответственно, $h_{l s}-$ энтальпия жидкости на линии насыщения, $N_{b}=q /\left(\rho_{w r}\right)$ - число кипения, $q$ - тепловой поток, $r$ - удельная теплота парообразования, $\pi=P / P_{c r}-$ относительное давление, $P_{c r}-$ критическое давление, $g$ - ускорение земного тяготения. Среднюю плотность жидкости $\rho_{l, j}$ определяют как $\rho_{l, j}\left(h_{l, j}\right)$ в зависимости от энтальпии жидкости в сечении $j$ : $h_{l, j}=h_{j}-r x_{j}$. Теплофизические свойства теплоносителя можно выбрать по таблицам из работы [16].

В представлении экспериментальных результатов по гидродинамике в обогреваемых каналах в качестве независимых переменных используют среднее значение по длине измерительного участка относительной энтальпии $\left\langle x_{r}>\right.$ или истинного паросодержания $\langle x\rangle$. Такое использование этих величин подразумевается в дальнейшем и поэтому обозначение осреднения опускается.

На рисунках 1 и 2 представлены характерные зависимости потерь давления от относительной энтальпии $x_{r}$ для вертикального канала в виде обогреваемой трубы. Гидравлическое сопротивление в канале начинает активно расти с момента начала парообразования, достигает максимума, а затем уменьшается до величины, характерной для течения пароводяной смеси при высоком паросодержании. Последние точки на рисунках соответствуют потерям непосредственно перед кризисом теплоотдачи.

Во всем диапазоне исследованных режимов поведение потерь давления $\triangle P$ остается монотонным с характерным максимумом при значениях относительной энтальпии в диапазоне $0.6 \ldots 0.8$ независимо от величины массовой скорости и давления. В опытах не обнаружено и появление «кризиса сопротивления» с двумя экстремумами как это показано в работах [5-8].

Представим потери давления в области двухфазного течения $\Delta P_{2 \phi}$ в форме уравнения (1), но как разность между полученным значением $\Delta P$ по уравнению (2) и потерями $\Delta P{ }_{i}$, которые соответствуют началу парообразования в канале:

$\frac{\Delta P_{2 \Phi}}{\Delta Z}=\frac{\Delta P-\Delta P_{i}}{\Delta Z}=\Phi(x) F(\rho w) F(P) F(q) F(D) \frac{(\rho w)^{2}}{2 \rho^{*} D}$,

где $\Phi(x)$ - некоторая функция от паросодержания. В этом случае при рассмотрении потерь давления в зависимости от массового паросодержания примем очевидное условие $\Delta P_{2 \phi}=0$ при $x_{r}=x_{i}$ и, соответственно, $\Delta P_{2 \phi}=0$ при $x=0$. Минимальное значение потерь дав- 
ления $\Delta P_{i}$ в уравнении (3) определим по соотношению:

$$
\Delta P_{i}=\xi_{i} \frac{(\rho w)^{2}}{2 \rho_{i}} \frac{\Delta Z}{D},
$$

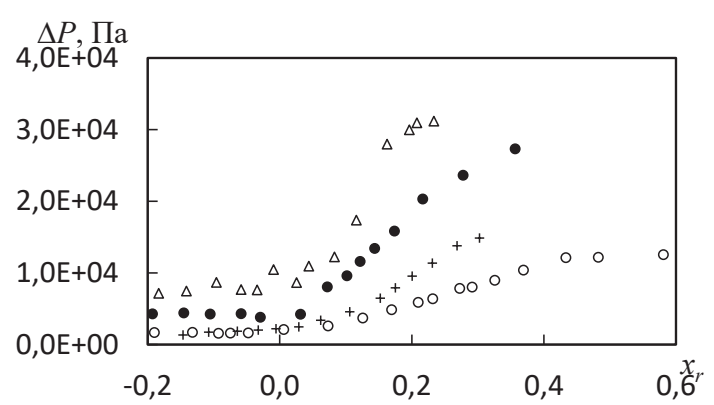

Рис. 1. Изменение гидравлического сопротивле-ния от относительной энтальпии для трубы $D=0.01, \Delta Z=0.635, P=7: \Delta-\rho w=3007$; $\bullet-2021 ;+-1500 ; \circ-1005$.

Теплофизические свойства воды определены по среднемассовой энтальпии в сечении начала парообразования (скорректированное соотношение из работы [15]):

$$
h_{i}=h_{l s}-370 \frac{q}{\rho w}(1+0.63 \pi) .
$$

В отличие от адиабатного течения двухфазного теплоносителя переменная $x$ в уравнении (1) является функцией от теплового потока, массовой скорости и давления. Поэтому представление потерь в виде произведения влияющих функций от режимных параметров можно рассматривать как произведение поправок к основной зависимости $\Delta P_{2 \phi}(x(q, \rho w, P))$. В представленном здесь исследовании использовались только трубы из нержавеющей стали с технической шероховатостью при вертикальном их расположении и поэтому в уравнениях отсутствуют соответствующие поправки. Однако в общем случае такие поправки могут присутствовать.

При сравнении результатов исследований потерь давления в канале с подводом и без подвода тепла возникает необходимость определения степени влияния теплового потока на поведение кривой $\Delta P_{2 \phi}(x)$. Поскольку

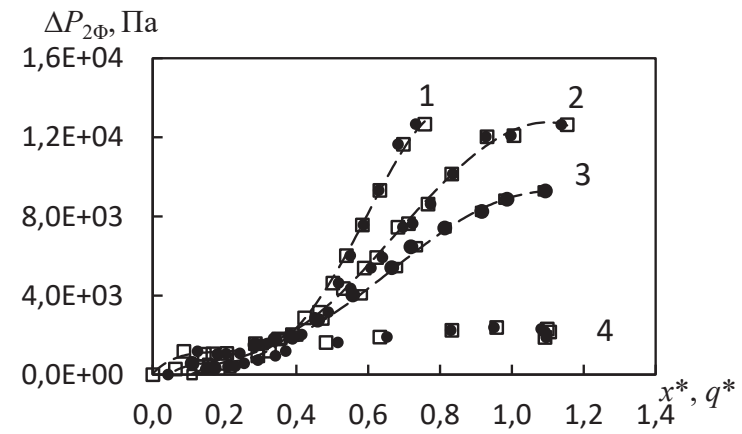

Рис. 3. Изменение гидравлического сопротивления канала при переменном тепловом потоке:

$D=0.01, Z=0.635, P=7: 1-\rho w=1500 ; 2-\rho w=1005$;

$$
3-\rho w=770 ; 4-P=10, \rho w=366 ;
$$$$
\bullet-\Delta P_{2 \Phi}(x *) ; \square-\Delta P_{2 \Phi}(q *) \text {. }
$$

где для вычисления коэффициента сопротивления $\xi_{i}$ использована формула Филоненко:

$$
\xi_{i}=\left(1.82 \lg \operatorname{Re}_{i}-1.64\right)^{-2} .
$$

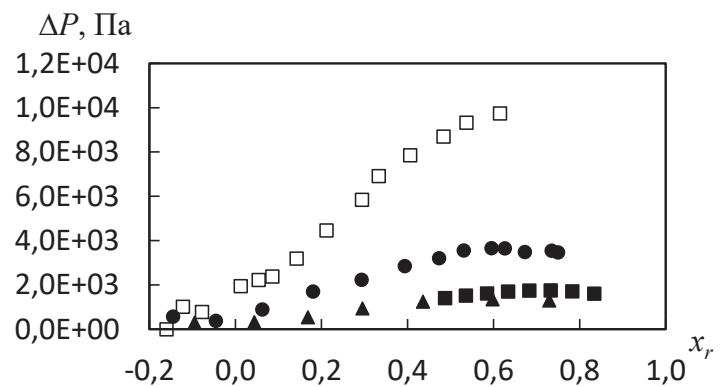

Рис. 2. Изменение гидравлического сопротивления от относительной энтальпии для трубы

$D=0.01, \Delta Z=0.635 ; \square-P=7, \rho w=770$;

$\bullet-P=10, \rho w=500 ; \Delta-P=14, \rho w=325$;

$D=0.013, \Delta Z=0.325:-P=4, \rho w=300$.

в диабатном течении переменная х зависит от $q$, тогда влияние этого параметра на гидравлическое сопротивление можно определить непосредственно в опытах с переменным тепловым потоком при постоянном давлении и массовой скорости. Для определения поправки $F(q)$ в уравнении (3) представим зависимость потерь для некоторых режимов, показанных на рисунках 1 и 2, в координатах $\left[\Delta P_{2 \phi} ; x^{*}\right]$ и $\left[\Delta P_{2 \phi} ; q^{*}\right]$. Для этого обозначим $x^{*}=x_{r}-x_{i}$ и преобразуем переменную $q$ к безразмерному виду $q^{*}$ так, чтобы при условии $q=q_{i}$ в сечении начала кипения при $x_{r}=x_{i}$ выполнялось $q^{*}=0$ : $q^{*}=C\left(q-q_{i}\right) / q_{i}$, где $C$ - постоянная для выбранного режима. Совпадение кривых $\Delta P_{2 \phi}\left(x^{*}\right)$ и $\Delta P_{2 \phi}\left(q^{*}\right)$, показанное на рисунке 3 , можно получить и для любого другого случая. Это свидетельствует о том, что функция $\Delta P_{2 \phi}(x)$ инвариантна относительно $q$. Следовательно, поправка $F(q)$ в уравнении (3) равна единице. Таким образом, если потери давления в зависимости от паросодержания представлены уравнением (3), тогда этим в полной мере учтено влияние теплового потока и нет необходимости в его дополнительной коррекции.

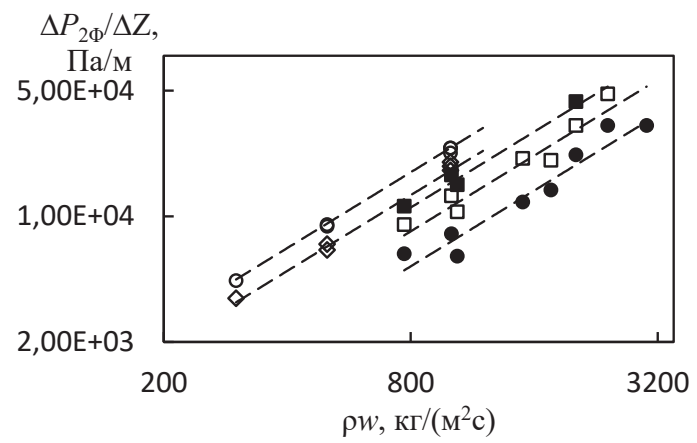

Рис. 4. Влияние массовой скорости на гидравлическое сопротивление в обогреваемом канале:

$D=0.013, P=4: \circ-x=0.4 ; \diamond-x=0.3 ; D=0.01$, $P=7: \square-x=0.4 ; \square-x=0.3 ; \bullet-x=0.2$; линии - функция $а(\rho w)^{1.4}$. 
На рисунке 4 показано влияние массовой скорости на гидравлическое сопротивление в опытах на трубе при постоянном давлении и массовом паросодержании. Разброс значений показателя степени $n$ в уравнениях для осредняющих кривых $a(\rho w)^{n}$ при постоянных коэффициентах $a$ составляет $n=1.4 \pm 0.04$, что соответствует результатам, полученным ранее авторами работ $[10,12]$. Сохраняя форму уравнения (3), представим соответствующую поправку на массовую скорость через число Рейнольдса для течения двухфазной смеси как:

$$
F(\rho w)=\left(\frac{\rho w D}{\mu_{m}}\right)^{-0.6}=\operatorname{Re}_{m}^{-0.6},
$$

где выбор коэффициента вязкости смеси $\mu_{m}$ является достаточно произвольным. При исследованиях потерь давления минимальная величина средней относительной энтальпии в измерительном участке чаще всего незначительно отличается от границы перехода к развитому кипению. Поэтому при выборе соотношения для $\mu_{m}$ вполне допустимо использовать уравнение MacAdams'a [17], в котором вязкость жидкости $\mu_{l s}$ выбрана при температуре насыщения:

$\frac{1}{\mu_{m}}=\frac{x \mu_{l s}+(1-x) \mu_{g}}{\mu_{l s} \mu_{g}}$.

Использование числа Рейнольдса в поправке на массовую скорость неизбежно связывает ее с поправкой на

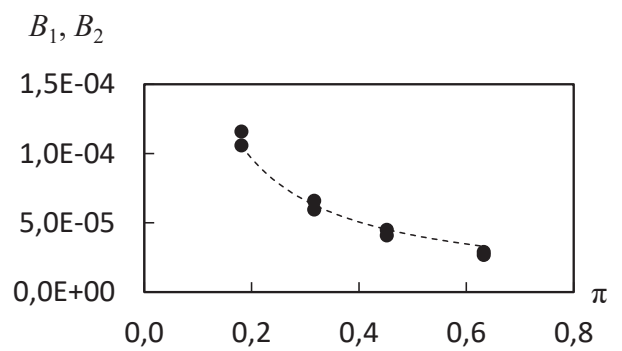

Рис. 5. Влияние давления на гидравлическое сопротивление: $\bullet-$ комплекс $B_{1}=\mu_{m}^{0.6} / \rho_{g} \pi^{-1.09}$ для $\boldsymbol{x}=0.3$ и $\boldsymbol{x}=0.4 ;$ пунктирная линия опытные данные для $B_{2}=k \Delta P_{20} / \Delta Z \sim \pi^{0.93} n р и$ $x=0.3$ u $\rho w=1000$.

При описании опытных данных по гидравлическому сопротивлению традиционно используют представление потерь в зависимости от паросодержания в канале. Но в корреляционных уравнениях функция от паросодержания не всегда присутствует в явном виде. Тем не менее результаты в виде графиков для $\Delta P_{2 \phi}(x)$ показывают, что функция $\Phi(x)$ является неразрывнои в диапазоне $0 \leq x \leq x_{c r}$ и имеет один максимум в области высоких паросодержаний. Из уравнения (3) с учетом найденных поправок представим эту функцию в виде:

$\Phi(x)=\frac{\Delta P_{2 \Phi}}{\Delta Z} \operatorname{Re}_{m}^{0.6}\left(\frac{D_{10}}{D}\right)^{0.6} \frac{2 \rho_{g} D}{(\rho w)^{2}}$.

График изменения $\Phi(x)$ показан на рисунке 6 для экс- давление через вязкость смеси. Кроме этого влияние давления на гидравлическое сопротивление определяется тем, какая плотность $\rho^{*}$ введена в уравнение (3). Во многих случаях $[5 \ldots 8,10]$ для этого используют плотность жидкости при температуре насыщения $\rho_{l s}$ или среднюю плотность смеси [9]. Однако, при исследовании потерь давления в обогреваемой трубе с внутренним диаметром $D=0,013$ [11] было обнаружено, что в диапазоне изменения давления от 4 до 14 МПа гидравлическое сопротивление изменяется приблизительно обратно пропорционально приведенному давлению $\pi=$ $P / P_{c r}: \Delta P_{2 \phi} / \Delta Z \sim \pi^{-1}$. В то же время изменение комплекса $B_{1}=\mu_{m}^{0.6} / \rho_{g}$ в зависимости от давления подчиняется такому же соотношению. Следовательно, если в уравнении (3) в качестве $\rho^{*}$ использовать плотность $\rho_{g}$, тогда с учетом влияния вязкости по соотношению (4) ${ }^{\prime}$ это приведет к приближенному равенству $F(P) \approx 1$. Рисунок 5 показывает, что с допустимой погрешностью такое приближение имеет смысл использовать.

Использование числа $\mathrm{Re}_{m}$ в поправке $F\left(\rho_{w}\right)$ приводит к необходимости введения поправки на диаметр канала: $F(D)=\left(D / D_{10}\right)^{0.6}$. Здесь в качестве нормированного значения гидравлического диаметра $D_{10}$ выбрана труба с внутренним диаметром 0.01 м. Опыты с трубопроводами (гидравлический диаметр составлял 0.01 и 0.013 м) и кольцевыми каналами (гидравлический диаметр 0.004 м) показали, что такая поправка удовлетворительно описывает полученные результаты.

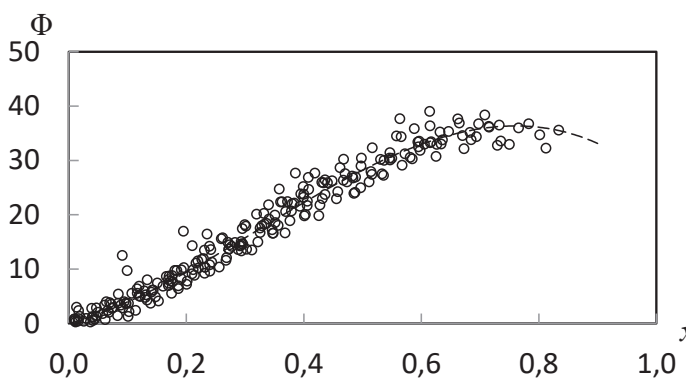

Рис. 6. Функция влияния паросодержсания на коэффициент гидравлического сопротивления в обогреваемом канале. Опытные данные соответствуют режимным параметрам из таблицы. Пунктирная линия - по уравнению (6).

периментальных данных, полученных в трубах и кольцевых каналах в исследованной области изменения параметров. Плотность расположения данных позволяет с приемлемой ошибкой выделить осредняющую кривую. С погрешностью, не превышающей $\pm 25 \%$, опытные точки группируются около кривой в виде полинома:

$\Phi(x)=C\left(k_{1} x^{3}+k_{2} x^{2}+k_{3} x\right)$ при $0 \leq x \leq x_{c r}$.

Наиболее вероятными значениями коэффициентов после определения всех поправок являются: $C=114$, $k_{1}=-1, k_{2}=0.97, k_{3}=0.26$. В этом случае, как не трудно убедиться, максимальное значение функции $\Phi(x)$ находится при паросодержании $x \approx 0.78$. Расчеты по методу Martinelli R.C [14] для расслоенного адиабатного тече- 
ния дают более высокое значение паросодержания, при котором наблюдается максимум потерь давления. Экспериментальное определение коэффициентов в уравнении (6) и, соответственно, положения точки перегиба $\Phi(x)$ зависит от величины приращения паросодержания на измерительном участке. Стремление к уменьшению относительной длины $\Delta Z / D$ с целью достижения более высоких средних значений $\langle x\rangle$ ведет к росту погрешности измерений перепада давления. Значения коэффициентов в уравнении (6) получены для условий, когда максимальное приращение относительной энтальпии на измерительном участке составляло $0.1 \ldots 0.3$. Уточнение координаты точки перегиба возможно при получении опытных данных для таких режимов, когда критическое паросодержание оказывается в области $x_{c r}>0.9$, а приращение паросодержания на участке не превосходит 0.1, что связано с известными трудностями. Тем не менее, уравнение (6) для $\Phi(x)$ с указанными коэффициентами хорошо обобщает экспериментальные данные для широкого диапазона изменения режимных параметров до кризиса теплоотдачи. Потери давления в вертикальном парогенерирующем канале с учетом (6) можно записать в виде соотношения:

$\Delta P=\left[\xi_{i} \frac{\rho_{g}}{\rho_{i}}+\frac{\Phi(x)}{\operatorname{Re}_{m}^{0.6}}\left(\frac{D}{D_{10}}\right)^{0.6}\right] \frac{\Delta Z}{D} \frac{(\rho w)^{2}}{2 \rho_{g}}$,

$\Phi(x)=114\left(-x^{3}+0.97 x^{2}+0.26 x\right)$ при $0 \leq x \leq x_{c r}$.

Полученное уравнение пригодно для расчета потерь давления при кипении воды в трубах и кольцевых каналах в диапазоне изменения режимных параметров, указанных в таблице 1. Из 720 опытных данных 89 \% точек не выходят за пределы относительной погрешности $\pm 25 \%$.

Иногда описание потерь давления разбивают на две зоны в зависимости от термодинамического состояния потока $[9,17,19]:$ течение с пузырьковым кипением недогретой до температуры насыщения воды и дисперсно-кольцевой режим течения, когда среднемассовая температура потока равна температуре насыщения. Однако представленные результаты опытов не дают оснований для определения каких-либо граничных условий для перехода между зонами и поэтому такое разбиение является чисто формальным. Для расчета потерь давления с той же погрешностью $\pm 25 \%$ при $x \leq 0.2$ можно использовать упрощенную функцию $\Phi(x)=65 x^{1.2}$. Тогда уравнение (7) можно переписать в виде:

$\frac{\Delta P}{\Delta Z}=\left[\xi_{i} \frac{\rho_{g}}{\rho_{i}}+\frac{65 x^{1.2}}{\operatorname{Re}_{m}^{0.6}}\left(\frac{D}{D_{10}}\right)^{0.6}\right] \frac{(\rho w)^{2}}{2 \rho_{g} D}$ при $0 \leq x \leq 0.2$.

Опытные данные для кольцевых каналов, как это видно из таблицы, находятся в области паросодержаний $x<0.25$ и, соответственно, в области низких абсолютных значений. Поэтому они укладываются в принятое описание по уравнению (8) с приемлемой относительной погрешностью.

\section{Сравнение полученных соотночений с известныли корреляциями}

Наиболее известные расчетные методики дают возможность построить картину поведения потерь давления в каналах при течении двухфазной смеси в зависимости от паросодержания потока. Корреляция Боришанского В.М.[10] и полученное соотношение (7) содержат функцию влияния паросодержания $\Phi(x)$ в явном виде, что существенно упрощает анализ поведения гидравлического сопротивления. В других методиках $[7,8,19]$ такая функция присутствует в неявном виде и тогда сравнительный анализ возможен при расчете потерь давления для выбранных условий получения опытных данных.

Корреляция Боришанского В.М. и полученное соотношение (7) не содержат функцию влияния теплового потока и поэтому пригодны для сравнения с результатами, полученными как в адиабатных условиях, так и в условиях с подводом тепла.

Сравнение с расчетом по методу Martinelli R.C. [4] представим с учетом соотношений для числа Рейнольдса и градиента давления при турбулентно-турбулентном режиме течения:

$$
\operatorname{Re}_{g}=\frac{x \rho w D}{\mu_{g}} ; \frac{d P_{g}}{d Z}=\frac{0.186}{\operatorname{Re}_{g}^{0.2}} x^{1.8} \frac{(\rho w)^{2}}{2 \rho_{g} D} .
$$

Параметр двухфазности $\Phi_{g}\left(X_{t}\right)$ определим из номограммы [14] по параметру $X_{t t} \stackrel{g}{=}\left[d P_{1} / d P_{g}\right]^{0.5}$. В результате получим выражение для вычисления потерь давления [4]:

$$
\frac{\Delta P}{\Delta Z}=\frac{0.186}{\operatorname{Re}_{g}^{0.2}} x^{1.8} \Phi_{g}^{2}\left(X_{t t}\right) \frac{(\rho w)^{2}}{2 D \rho_{g}} .
$$

Представленные на рисунке 7 данные показывают, что потери давления для массовой скорости менее 1000 кг/(м² c), рассчитанные по (9) для давлений менее 7 МПа хорошо согласуются с опытными данными. Однако с ростом давления наблюдаются значительные отклонения расчета по (9) от опытных данных и от расчета по (7), что связано с изменением теплофизических свойств теплоносителя, которые по-разному отражаются в методиках. Не является неожиданностью то, что с ростом массовой скорости отклонения результатов по (9) увеличиваются (рис. 8) по сравнению с опытными данными. Методика (9) предусматривает использование коэффициентов сопротивления в форме Блазиyса с показателем степени $n=-0.2$ при числе Re, a не $n=-0.6$, полученном в (7). Ранеe Wallis G.B. [14] на примере опытных данных для давления $~ 7$ МПа показал, что с увеличением массовой скорости экспериментальные данные отклоняются от результатов расчета по методу Martinelli R.C. в сторону гомогенной модели.

Уравнение (1) Боришанского В.М. с сотр. [10] получено для пучков стержней, но авторы пред-лагают использовать его и для труб при условии $F(D)=1$, что предполагает отсутствие влияния диаметра канала на коэффициент гидравлического сопротивления:

$\Delta P=\left\{\xi_{0}+\frac{0.316 x}{1+x}[0.025+\right.$ 
$\left.\left.+1.7\left(1-\frac{P-1}{1.64-0.915(P-1)}\right)\right]\left(\frac{\rho w}{\rho_{l s}}\right)^{-0.6}\right\} \frac{(\rho w)^{2}}{2 \rho_{l s}} \frac{\Delta Z}{D}$,

где $P$ - давление, МПа, $\xi_{0}$ - коэффициент гидравлического сопротивления при течении насыщенной жидкости: $\xi_{0}=(1.82 \mathrm{lg} \mathrm{Re}-1.64)$. В уравнение введена независимая функция от паросодержания и поправка на давление в явном виде для области паросодержания $0 \leq x \leq 0.9$. Недостаток приведенного уравнения заключается в том, что оно применимо в ограниченном диапазоне изменения давления $4<P<12$ МПа, поскольку поправка, заключенная в квадратные скобки в уравнении

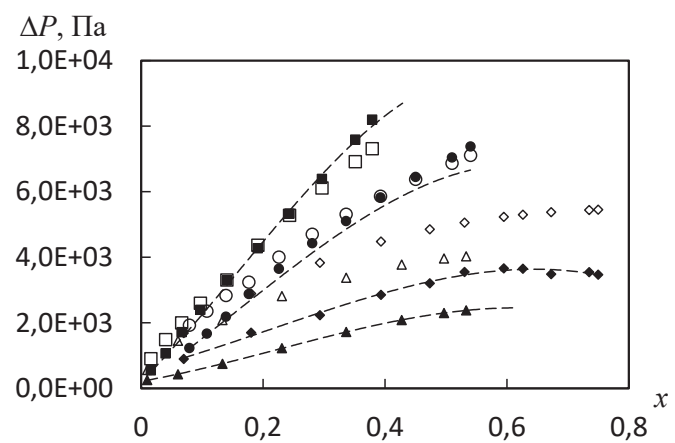

Рис. 7. Сравнение опытных данных (зачерненные точки) с расчетом по методике (7) (линии) и по методике (9) (незачерненные точки) для массовой скорости $\rho w=500$ при давлении:

घ, $\square-P=4 ; \bullet, \circ-P=6 ; \diamond, \diamond-P=10 ; \Delta, \Delta-P=14$.

ченных в ЦКТИ [5], и согласуется с уравнением (7) при паросодержаниях $x \leq 0.2$ :

$$
\begin{aligned}
& \psi=\left[1+x\left(\frac{\rho_{l s}}{\rho_{g}}-1\right)\right]\left[1+x\left(\frac{\mu_{l s}}{\mu_{g}}-1\right)\right]^{-0.2 x} \\
& \left\{1+0.57 x^{0.125}(1-x)^{2}\left[\left(0.2+\operatorname{Fr}_{0}^{0.5} \frac{\rho_{g}}{\rho_{l s}}\right)^{-1}-5.2 x^{2}\right]\right\} .
\end{aligned}
$$

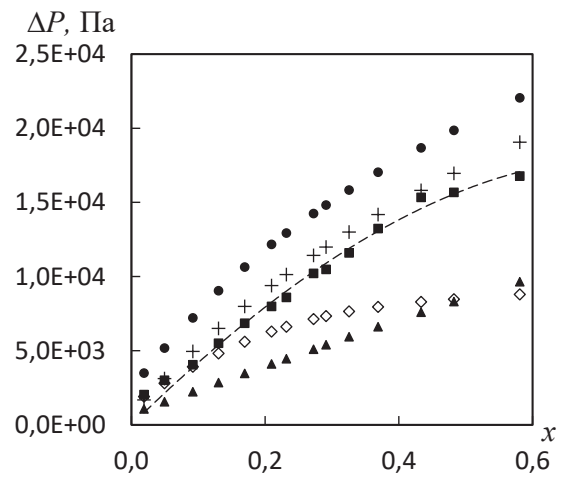

Рис. 9. Сравнение опытных данных с расчетом для массовой скорости $\rho w=1000$ и давления $P=7$ :

- - по методике (9); + - по формуле (10);

- опытные данные; $\diamond$ - по формуле (11);

$\triangle$ - по Букринскому А.M. [8]; линия-расчет по (7).
(10), терпит разрыв при давлении вблизи 3 МПа. Кроме того, введенная функция $F(x)=x /(1+x)$ является монотонно возрастающей в области больших паросодержаний $x$, что не соответствует опытным данным. Отсутствие влияния эквивалентного диаметра канала тоже не улучшает расчет по этой методике. Расчет по (10) дает лучшее согласование с экспериментальными данными по сравнению с методом (9) в области высоких скоростей и удовлетворительно совпадает с опытом при давлениях $\leq 7$ МПа для труб с гидравлическим диаметром $0.01 \ldots 0,013 \mathrm{M}$.

Уравнение Лысцовой Н.Н. [7] для поправки на негомогенность $\psi=\Delta P / \Delta P_{0}$ основано на данных, полу-

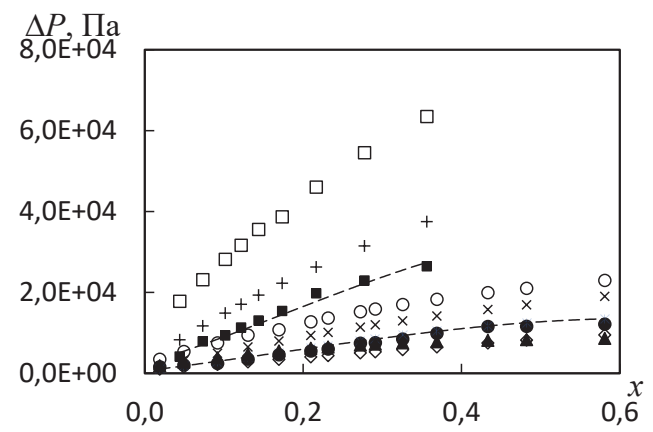

Рис. 8. Сравнение опытных данных (зачерненные точки) с расчетом по методике (7) (линии), по (9) (незачерненные точки) и по (10) (+, x) для давления $P=7$ и массовой скорости: $\bullet, \circ, x-\rho w=1005 ; \square, \square,+-\rho w=2021$.

При $x>0.2$ уравнение (11) дает существенно заниженные значения потерь давления.

В уравнении Букринского А.М. $[1,8]$ используются постоянные коэффициенты для фиксированных давлений, вследствие чего оно пригодно для описания частных опытных данных и его применение не может быть расширено. В этом уравнении влияние массовой скорости зависит от давления и меняется случайным образом от $(\rho w)^{1.3}$ до $(\rho w)^{1.8}$, что не подтверждается опытными данными. Например, для скоростей ниже и выше 1000, при давлении 70 бар приведенная поправка на негомогенность $\psi$ дает расходящиеся значения: для скорости 350 и при $x=0.5$ значение $\psi$ становится $\approx 0$, а для скорости 3000 при том же паросодержании увеличивается до 40. При этом экспериментальное значение и поправка (11) находятся в пределах 14 и 10, соответственно. На рисунке 9 приведено сравнение экспериментальных данных с расчетами по упомянутым методикам.

В работах Бартоломея Г.Г. с соавторами [3, 9, 19] представлены уравнения для потерь давления при кипении воды в трубах как для области неразвитого кипения $[3,9]$, так и для области равновесного двухфазного потока [19]. Оба уравнения дают одинаковый результат при расчете потерь в области изменения среднего на участке паросодержания $0 \leq x \leq 0.2$, который согласуется с экспериментальными данными. Для области развитого кипения результат расчета по предлагаемому соотно- 
шению мало отличается от расчета по (11), сходится с опытными данными только в области $x \leq 0.15$ для каналов с гидравлическим диаметром $D \leq 0.005$ м и приводит к значительным отклонениям от опытных данных для больших паросодержаний.

\section{Заключение}

Как показали исследования, описание потерь давления в обогреваемых каналах при вынужденном течении двухфазного теплоносителя можно представить в виде уравнения, которое содержит произведение функций от влияющих параметров. Такие функции являются инвариантными и могут быть определены экспериментально с достаточной точностью. Описание потерь давления в зависимости от паросодержания в потоке не содержит функцию влияния теплового потока в явном виде. При этом использование в уравнении свойств газа при температуре насыщения приводит к исключению функции влияния давления в явном виде. Потери давления имеют выраженный максимум при массовом паросодержании $\approx 0.7$, который не зависит от режимных параметров.

\section{ЛИТЕРАТУРА}

1. Петухов Б.С.,Генин Л.Г., Ковалев С.А. Теплообмен в ядерных энергетических установках // М.: Энергоатомиздат. 1986. С. 373.

2. Кириллов П.Л., Юрьев Ю.С., Бобков В.П. Справочник по теплогидравлическим расчетам (ядерные реакторы, теплообменники, парогенераторы). М.: Энергоатомиздат, 1990. С. 360.

3. VDI Heat Atlas Second Edition, Springer-Verlag Berlin Heidelberg. 2010

4. Chisholm D. Two-phase flow in pipelines and heat exchangers // London. - 1983. - P. 304.

5. Тарасова Н.В. Гидравлическое сопротивление при кипении воды и пароводяной смеси в обогреваемых трубах и кольцевых каналах // Тр. ЦКТИ. - 1965. Вып. 59. - С. $47-58$.

6. Тарасова Н.В., Леонтьев А.И., Трагова Л.А., Синяков И.И., Боронина Л.В. Гидравлическое сопротивление двухфазного потока применительно к условиям работы канального реактора.// М.: Энергия. - 1977. Труды ВТИ. - Вып. 11. - С. 202 - 213.
7. Osmachkin V.S., Borisov V.D. Pressure Drop and Heat Transfer for Flow of Boiling Water in Vertical Rod Bundles // Proc. IV Int. Heat Transfer Conf. - 1970. - B4.9.

8. Букринский A.M. Аварийные переходные процессы на АЭС с ВВЭР. - М.: Энергоиздат, 1983. - С. 142.

9. Бартоломей Г. Г., Коврижных В. П. Обобщение экспериментальных данных по гидравлическому сопротивлению при кипении с недогревом. - Теплоэнергетика, 1991, № 12 , с. $38-41$.

10. Боришанский В.М., Андреевский А.А., Чистяков В.А., Фромзель В.Н., Фокин Б.С., Данилова Г.П., Быков Г.C., Шлейфер В.A. Гидравлическое сопротивление при продольном обтекании пучков стержней пароводяным потоком / Достижения в области исследования теплообмена и гидравлики двухфазных потоков в элементах энергооборудования. - Л.: Наука, 1973. - С. $144-163$.

11. Aнтипов B.Г. Влияние паросодержания на гидравлическое сопротивление каналов // Вопросы атомной науки и техники. Сер. Ядерная техника и технология. - 1989. - Вып. 5. - С. 49 - 53.

12. Silvestri $M$. Fluid Mechanics and Heat Transfer of Two-Phase Annular-Dispersed Flow // Advances in Heat Transfer. - 1964. - V. 1. - P. $355-446$.

13. Дорошук B.E. Кризисы теплообмена при кипении воды в трубах. М.: Энергоатомиздат. 1983.

14. Уоллис Г. Одномерные двухфазные течения. М: Мир. - 1972. - 440 С.

15. Aнтипов В.Г. Паросодержание в области неравновесного кипения воды // Пром. теплотехника. - 2012. № 5. - С. $25-30$.

16. Александров А.А., Григорьев Б.А. Таблицы теплофизических свойств воды и водяного пара. М.: Издательство МЭИ. 1999. - 168 с.

17. McAdams W.H. et al., Trans. ASME, 64, 193 (1942).

18. Кириллов П.Л., Смогалев И.П., Дорошенко В.А., Суворов М.Я. Методика гидравлического расчета вертикального парогенерирующего канала // Теплоэнергетика. - 1980. - № 2. - С. $71-74$.

19. Бартоломей Г.Г., Дунаев Ю.А., Коврижных В.П., Харитонов Ю.В. Обобщение экспериментальных данных по гидравлическому сопротивлению при кипении воды в трубах // Тепломассообмен-ММФ-92. Т. 4. Ч. 1. Минск. 1992. С. $107-110$. 


\section{THE PRESSURE LOSSES IN A FORCED WATER FLOW IN THE STEAM-GENERATING CHANNELS}

\section{Antipov V.G.}

Scientific-production enterprise "PASKAL", vul. Zhelyabova, 2a, Kyiv, 03680, Ukraine

The known empirical correlation ratios for estimation of the drop of pressure in the steam generating channel have considerable usage limitations and vary in the amount. Generalization of the experimental data in the form of a correlation that includes the product of functions from the influencing variables yields a physically consistent outcome. Suchlike functions are invariant and can be made rather accurate. The research work introduces the experimental data on the fluctuation of the pressure losses for the twophase flow of water in the vertical pipe and the annular channel in the heat input conditions. The researches have been conducted within the range of change of the following parameters: the hydraulic diameter from 0.004 to $0.013 \mathrm{~m}$, the pressure from 4 to $16 \mathrm{MPa}$, the mass velocity from 200 to $3000 \mathrm{~kg} /\left(\mathrm{m}^{2} \mathrm{~s}\right)$, and the heat flow to $2.4 \mathrm{MW} / \mathrm{m}^{2}$. The researches have proved that the dependence of the pressure drop on the steam content is not influenced by the heat flow from the wall, and the influence of pressure is determined merely by the thermal-physical properties of the steam. The power function with the value of 1.4 proves the dependence of the pressure loss on the mass velocity. The correlation ratio for assessment of the pressure losses in the area of boiling to the point of burnout has been introduced. The experimental data have been compared to the commonly known assessment methods. It is advisable to use the research results in the thermohydraulic calculations of the elements of power generating units.

References 19, tables 1, figures 9.

Key words: water, boiling, pressure losses, vertical channel. 1. Petukhov B.S., Genin L.G., Kovalev S.A. Heat transfer in nuclear power plants. M.: Energoatomizdat. 1986. P.373. (Rus.)

2. Kirillov P.L., Yuryev U.S., Bobkov V.P. Handbook of thermal-hydraulic calculations (nuclear reactors, heat exchangers, steam generators). M.: Energoatomizdat. 1990. P.360. (Rus.)

3. VDI Heat Atlas Second Edition, Springer-Verlag Berlin Heidelberg. 2010

4. Chisholm D. Two-phase flow in pipelines and heat exchangers . London. 1983. P.304.

5. Tarasova N.V. Pressure drop at boiling water and steam- water mixture in the heated pipes and annular channels. Trudy CKTI. 1965. V. 59. P. 47 - 58. (Rus.)

6. Tarasova N.V., Leontyev A.I., Tragova L.A., Sinyakov I.I., Boronina L.V. Pressure drop of two-phase flow in relation to the conditions of the working channel of the reactor. Trudy VTI. M.: Energiya. 977. V. 11. P. 202 - 213. (Rus.)

7. Osmachkin V.S., Borisov V.D. Pressure Drop and Heat Transfer for Flow of Boiling Water in Vertical Rod Bundles. Proc. IV Int. Heat Transfer Conf. 1970. B4.9.

8. Bukrinskyi A.M. Emergency transients at nuclear power plants with WWER . M.: Energoizdat. 1983. P.142. (Rus.)

9. Bartolomei G.G., Kovrizhnykh V.P. Correlation of experimental data on hydraulic resistance with subcooled boiling. Teploenergetika. 1991. № 12. P.38 - 41. (Rus.)

10. Borishanskiy V.M., Andreyevsky A.A., Chistyakov V.A., Fromzel V.N., Fokin B.S., Danilova G.P., Bikov G.S., Shleifer $V . A$. Pressure Drop in longitudinal wrapping bundles of rods of steam-water flow. Advances in the study of heat transfer and hydraulics of two-phase flows in the elements of power, L.: Nauka. 1973. (Rus.)

11. Antipov V.G. The effect of steam quality on the pressure drope of the channels. Voprosy atomnoy nauki i tekhniki. Ser. Yadernaya tekhnika i tekhnologiya. 1989. V. 5. P.49 53. (Rus.)

12. Silvestri $M$. Fluid Mechanics and Heat Transfer of TwoPhase Annular-Dispersed Flow. Advances in Heat Transfer. 1964. V. 1. P.355 - 446.

13. Doroschuk V.E. Heat transfer crises during boiling of water in the pipes. M.: Energoatomizdat. 1983. P.142. (Rus.) 14. Wallis, G.B. One-Dimensional Two-Phase Flow. New York, McGraw-Hill. 1969. P.408.

15. Antipov V.G. Vapor Void Fractions in the Subcooled Boiling Region // Promyshlennaya teplotekhnika. 2012. № 5. P. 25 - 30. (Rus.)

16. Aleksandrov A.A., Grigoryev B.A. The tables of thermophysical properties of water and steam. M.: MEI. 1999. P.168 (Rus.)

17. McAdams W.H. et al., Trans. ASME, 64, 193 (1942).

18. Kirillov P.L., Smogalev I.P., Doroshenko V.A., Suvorov M.Y. Hydraulic calculation technique of vertical steam generating channel. Teploenergetika. 1980. № 2. P.71 - 74 . (Rus.)

19. Bartolomei G.G., Dunayev U.A., Kovrizhnykh V.P., Kharitonov U.V. Correlation of experimental data on hydraulic resistance in boiling water in the tubes. Heat/ Mass Transfer-MIF-92. V. 4. C. 1. Minsk. 1992. P.107-110. (Rus.) 